\title{
Low Molecular Weight Heparin versus
} Coumadin-Induced Skin Cecrosis in a Patient with Superficial Vein Thrombosis - A Case Report

\author{
Sala Nicoleta, Burdun Oana-Cristiana*, Botoman Maria, Rab Andrea, Bancu Ligia Ariana, Ureche Corina
}

University of Medicine and Pharmacy Tîrgu-Mures, Romania

\begin{abstract}
Introduction: Coumadin and low molecular weight heparin $(\mathrm{LMWH})$ induced skin necrosis are rare clinical conditions. Case presentation: A 63-years-old female patient, known with hypertension and right-sided hemiparesis, had presented to the emergency departement with pain and erythema of her internal right thigh. Venous Doppler-echography showed internal safenous vein thrombosis, thus she was started on anticoagulant treatment with Dalteparin and Acenocoumarol. On the 5th day of treatment she developed plantar hematoma on her left leg, followed by the appearance of hematomas, on the lower third of her right calf and on coxofemoral regions. Therefore, we raised the suspicion of heparin or coumadin-induced cutaneous necrosis, we stopped the anticoagulation for 5 days and restarted it with the administration of Rivaroxaban, fresh frozen plasma, antibiotics and local, sterile, saline dressings. After four days the patient presented deep vein thrombosis on her left calf. Results and Discussion: Coagulation abnormalities were suspected, but infirmed by normal values of specific laboratory tests, such as Protein C (472.2\%), protein S (77.10\%), lupus anticoagulant (LA1-screening 53, 5s, LA-confirmation 38,0s). Hemogram showed normal platelet values. To exclude a malignancy, abdominal CT scan was performed, which revealed a right adnexal inomogenic cystic lesion, CA-125 $(6,5 \mathrm{U} / \mathrm{ml})$, requiring further gynecological investigations. For the treatment of skin lesions, the patient required necrectomy and skin graft application. Conclusion: Particularly, considering that the patient was initially anticoagulated with LMWH and coumadins simultaneously, the etiology of her cutaneous lesions remains uncertain.
\end{abstract}

Keywords: skin necrosis; low molecular weight heparin, coumadins

Received: 9 December 2014 / Accepted: 26 April 2015

\section{Introduction}

Coumadin and LMWH (low molecular weight heparin) induced skin necrosis are rare clinical conditions. Diagnosis of LMWH/coumadin induced skin necrosis is usually suspected clinically, but a skin biopsy may be performed. Histopathology shows death of the surface skin and sometimes clots or inflammation in small blood vessels of the deeper skin [1-3]. Tissue lesions caused by cumadins appear in 0,1 $\%$ of patients having oral anticoagulant treatment, most of them manifesting after 3-6 days of treatment [4]. Heparininduced skin necrosis following treatment with unfractionated heparin was first reported in 1973 and four LMWHs, namely fraxiparine, dalteparin, enoxaparin and tinzaparin sodium have been linked with the condition [5]. Heparin induced skin necrosis may occur at the injection site or at distant areas, debuting in 1-17 days after the initiation of injections, one of these forms being associated with thrombocytopenia [1-3]. In many cases heparin necrosis is due to an allergic immune reaction involving a complex of antibody, heparin, platelet factor 4 (PF4) and platelet. This should be tested for as it is important not to have further heparin if it is positive. This form of heparin necrosis is called 'heparin-induced thrombocytopaenia type II' [6].

\section{Case presentation}

A 63-years-old Caucasian female, known with hypertension and a right-sided hemiparesis after a hemorrhagic stroke (2009), has been presented to the emergency department with pain and erythema of her internal right thigh. Her anterior medication included Metoprolol $100 \mathrm{mg}$, Enalapril 20mg, Felodipine 10mg and Fenofibrate $140 \mathrm{mg}$. Blood tests revealed high level of D-dimers and the Venous Doppler-echography showed internal safenous vein thrombosis, she was admitted in our clinic. After admittance anticoagulant (Dalteparin 7500UI 2x1 subcutaneous and Acenocoumarol $4 \mathrm{mg} 3 / 4 \mathrm{tb}$ oral) and antialgic treatment has been initiated.

On day 5 of anticoagulant treatment she presented a plantar hematoma on her left leg. On the 7 th day of hospitalization she developed hematomas with central necrosis on the lower third of her right calf and bilateral on coxofemoral regions. (Figure 1) The soft tissue ultrasound describes hematomas and the plastic surgeon diagnosed skin necrosis in the same regions. Considering these, a suspicion of heparin or coumadin-induced cutaneous necrosis has been raised, therefore the anticoagulant therapy was stopped and fresh frozen plasma and local sterile saline dressings were administrated on the skin lesions. On day 12 we restarted the anticoagulation with Rivaroxaban $15 \mathrm{mg} 2 \times 1$ oral. Due to clinical aspects of the skin lesions and according to plastic surgeon advice, Ceftriaxone has 


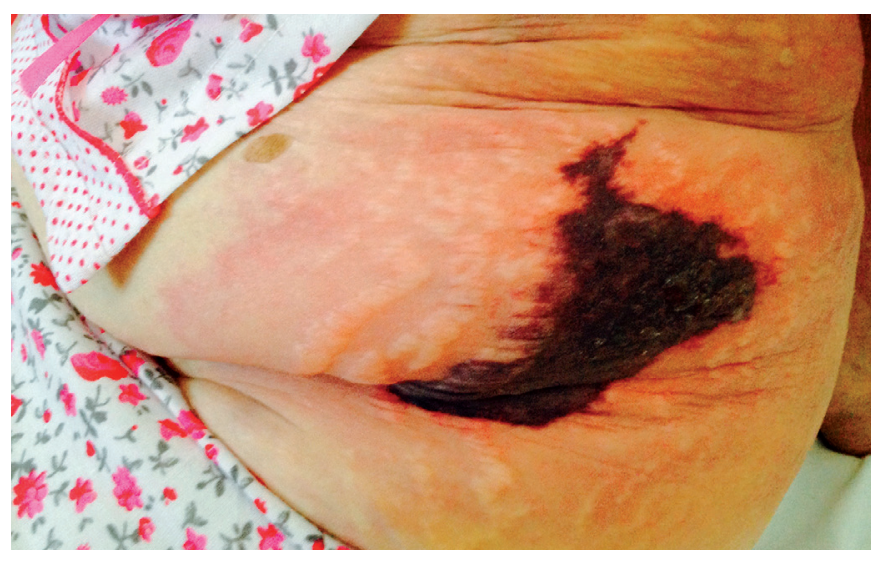

Fig. 1. Hematomas with central necrosis

been added as prophylaxis against lesions infection. On day 15 the patient accused pain, edema and erythema of left calf. The venous Doppler ultrasound of her left lower limb revealed deep vein thrombosis (DVT) of popliteal vein, posterior tibial vein, femoral vein, fibular vein and thrombosis of external saphenous vein. (Figure 2). The next day she developed fever $\left(38.2^{\circ} \mathrm{C}\right)$. We added a new i.v. antibiotic, Clindamycin, and the subsequently blood culture result was negative. We considered that the fever appeared as a consequence of skin necrosis.

\section{Results and Discussions}

Complete blood count revealed normal platelets values excluding the thrombocytopenic form of heparin induced skin necrosis. Hemoglobin levels decreased after the developing of new hematomas (11.2-9.8 g/dl). Because our patient had multiple-vein thrombosis and an atypical evolution of her disease under anticoagulants we evaluated the possibility of an antiphospholipid syndrome: we performed anticardiolipin IgG $(3.7 \mathrm{UI} / \mathrm{ml})$ and lupus anticoagulant (LA1-screening 53.5s, LA-confirmation 38,0s) and we found normal values. Coagulation abnormalities were suspected, but infirmed by specific laboratory tests, such as Protein S (77.10\%)-normal level and Protein C (472.2\%) - high level. A vasculitis was excluded by normal values of cryoglobulins, anti-dsDNA antibodies and circulating immune complexes. To exclude a malignancy as a cause of procoagulant status we done a chest X-ray and an abdominal CT-scan that describes right adnexal inhomogeneous cystic lesion. The gynecologist consultation put the diagnosis of benign right ovarian tumor in observation and CA-125 (6,5U/ml) had normal value. On hospital discharge we recommended to continue the anticoagulation with Rivaroxaban 10mg 2x1 oral, with a Veins Doppler

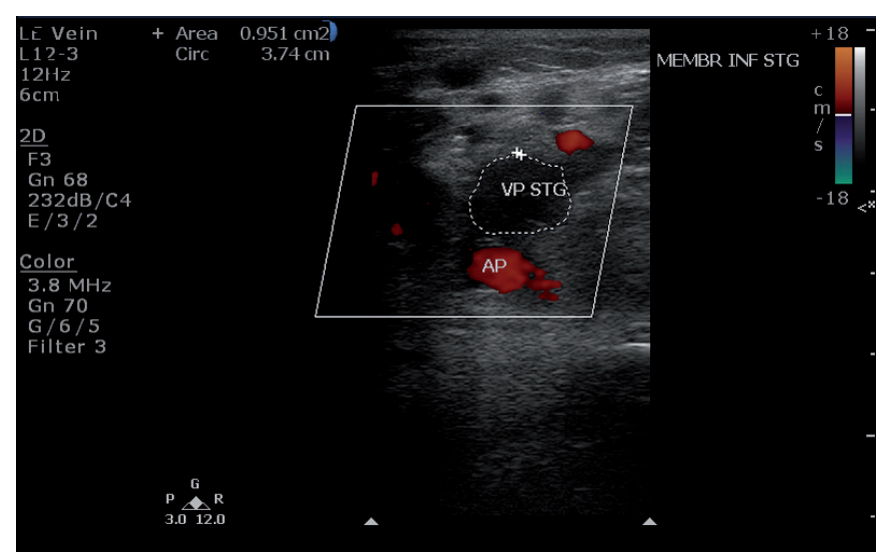

Fig. 2. Venous Doppler ultrasound revealing deep vein thrombosis of popliteal, posterior tibial and femoral vein and thrombosis of external saphenous vein

ultrasound reevaluation after 3 months. Plastic surgeon recommended necrectomy and skin graft application for the treatment of skin lesions. Gynecological reevaluation is necessary for the right ovarian tumor observation.

\section{Conclusion}

The particularity of this case is that a female patient, on anticoagulant treatment (Dalteparin and Acenocoumarol) for superficial vein thrombosis presented after 5 days skin hematomas with necrosis without thrombocytopenia. For this reason the classic anticoagulation has been stopped for 5 days and restarted with Rivaroxaban as a new therapy for DVT according to new data from literature. On the 4th day of Rivaroxaban our patient presented new DVT, the meaning of this fact could be the perturbation of coagulation present particularly in that patient or the lack of efficacy of the applied therapy.

Taking in consideration that the patient has been initially treated with LMWH and coumadin simultaneously, the etiology of her cutaneous lesions remains uncertain.

\section{References}

1. Handschin AE, Trentz O, Kock HJ, Wanner GA. Low molecular weight heparin-induced skin necrosis - a systematic review. Langenbecks Arch Surg, 2005;390: 249-254

2. Khan Z, Watson DK. Heparin-induced skin necrosis. Brit J Obstet Gynae, 2000;107:1315-1316.

3. Nadir Y, Mazor Y, Reuven B, Sarig G, Brenner B, Krivoy N. A fatal case of enoxaparin induced skin necrosis and thrombophilia. Eur J Haematol, 2006;77:166-168.

4. Janice M. Beitz. Coumadin-Induced Skin Necrosis. Wounds, 2002;14:7.

5. Drew JP, Smith MJ, Milling MAP. Heparin-induced skin necrosis and low molecular weight heparins. Ann R Coll Surg Engl, 1999;81:266-269.

6. Warkentin TE, Roberts RS, Hirsh J, Kelton JG. Heparin-Induced Skin Lesions and Other Unusual Sequelae of the Heparin-Induced Thrombocytopenia Syndrome*: A Nested Cohort Study. Chest, 2005;127:1857-1861. 